\title{
The diagnosis of thoracic outlet syndrome
}

This article was published in the following Dove Press journal:

Journal of Vascular Diagnostics

3 October 2014

Number of times this article has been viewed

Mark Lewis'

Akash Prashar'

Andoni P Toms'

Matthew P Armon ${ }^{2}$

Paul N Malcolm'

'Department of Radiology,

${ }^{2}$ Department of Vascular Surgery, Norfolk and Norwich University

Hospital, Norwich, UK
Correspondence: Mark Lewis Department of Radiology, Norfolk and Norwich University Hospital, Colney Lane, Norwich, NR4 7UY, UK Email mark.lewis@nnuh.nhs.uk
Abstract: "Thoracic outlet syndrome" is a term that covers a range of conditions and abnormalities causing a variety of presentations and symptoms. In order to assess and treat this group of conditions, careful consideration needs to be given to individual presentations to tailor appropriate clinical and imaging tests. There is no single test available for the definitive diagnosis and assessment of this group of conditions. This article aims to present an up-to-date review with particular focus on the diagnosis and relevance of the different imaging modalities. Chest radiography allows identification of bony abnormalities and apical lung tumors. Ultrasonography is useful in cases of suspected acute thrombosis of the upper limb. Computed tomography combined with computed tomography angiography provides a useful overview of the anatomical and vascular structures. Magnetic resonance imaging and magnetic resonance angiography are now widely used methods of assessment for vascular thoracic outlet syndrome, allowing dynamic assessment of the thoracic outlet, with good soft tissue contrast allowing for visualization of a wider range of pathologies than other imaging methods.

Keywords: thoracic inlet syndrome, MRI, CT

\section{Introduction}

Diagnosing thoracic outlet syndrome (TOS) is clinically challenging. This is because it is not a single, clearly defined clinical entity and may present with a variety of neurovascular symptoms.

In order to understand TOS, it is necessary to appreciate the anatomy of the thoracic outlet. TOS is the clinical result of constriction or compression of neurovascular structures in the interscalene triangle, costoclavicular space, or retropectoral (coracopectoral) space (Figure 1). These spaces are in continuity from the cervical spine, extending into the superior mediastinum and up to the lateral border of the pectoralis minor muscle. These three continuous compartments are also called the thoracic inlet, and this condition may also be referred to as "thoracic inlet syndrome". Several structures can give rise to the symptoms experienced and there are a large range of clinical presentations. In the early part of the 20 th century, the condition scalenus anticus syndrome was described and referred to compression of the subclavian artery in the thoracic outlet. ${ }^{1}$ In $1956,{ }^{2}$ the term "thoracic outlet syndrome" was coined by Peet et al and was described in relation to neurovascular symptoms.

This article aims to outline the background and anatomy of TOS and provide an up-to-date review of the clinical and imaging assessment of this condition. 

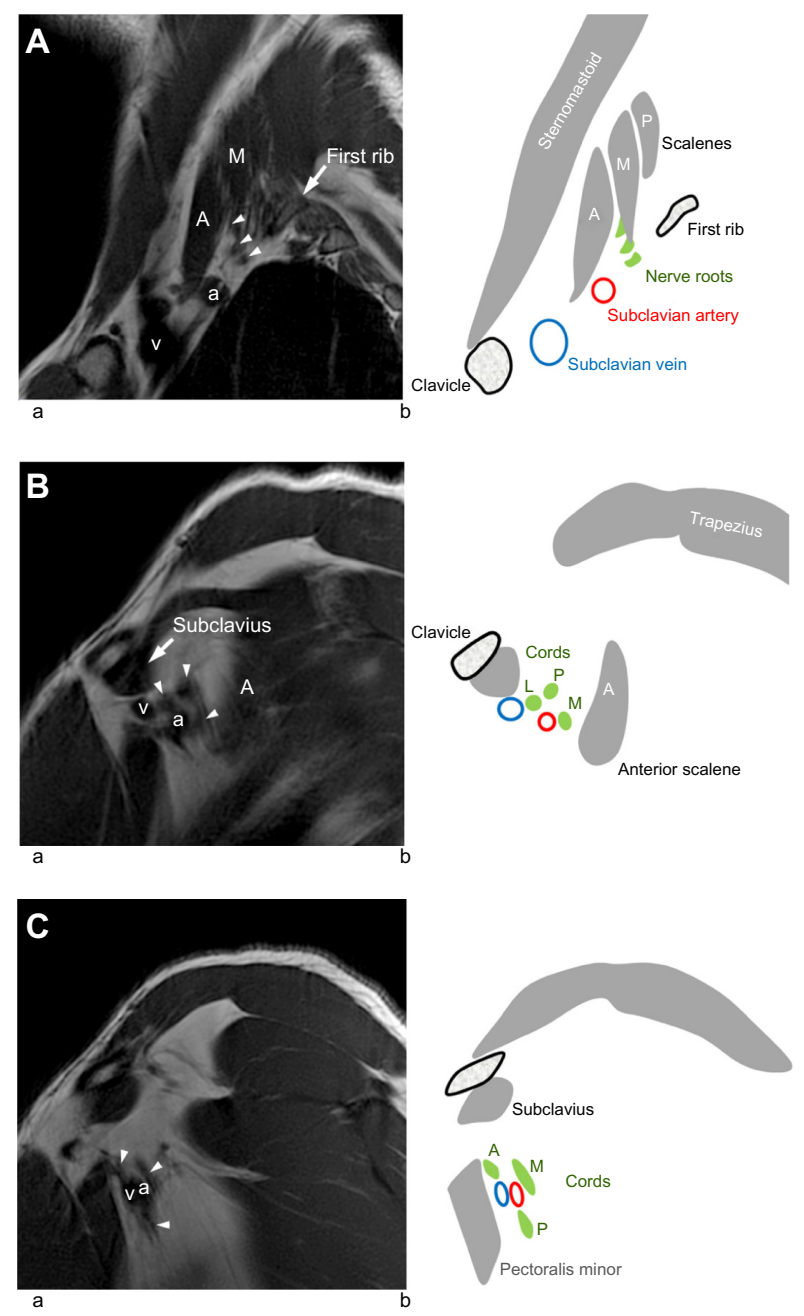

Figure I MRI and pictorial relationships of the anatomy of the thoracic outlet, divided into the interscalene triangle, the costoclavicular space, and the subcoracoid space. Notes: (A) MRI (a) and illustration (b) of the interscalene space, which is bordered by the anterior and middle scalene muscles anteriorly and anteroposteriorly, respectively, and the first rib infero-posteriorly. The roots of the brachial plexus lie above the subclavian artery. The subclavian vein is anterior to this space. (B) MRI (a) and illustration (b) of the costoclavicular space, which is bordered by the first rib inferiorly, the clavicle superiorly, and the subclavius anteriorly. The subclavian artery lies central with the vein anterior to it and branches of the brachial plexus are seen above and below the artery. (C) MRI (a) and illustration (b) of the subcoracoid (or retropectoralis minor) space, which is bordered by the pectoralis minor muscle anteriorly and the coracoid process superiorly. The cords of the brachial plexus can be seen around the vessels. Some compression of the vein is noted, and this is within normal variation. This space is lateral to the first rib and is least commonly involved in thoracic outlet syndrome. The arrowheads demonstrate the corresponding parts of the brachial plexus.

Abbreviations: A, anterior; M, medial; P, posterior; L, lateral; a, artery; v, vein.

\section{Etiology}

Anatomical conditions leading to TOS include an accessory cervical rib varying from a fully developed cervical rib (Figure 2) to a small or partially formed fibrous band. ${ }^{3}$ Movements of the arm can change the spaces within the thoracic outlet. Early functional studies with computed, tomography $(\mathrm{CT})$ and magnetic resonance imaging have shown the dynamic size and variability of these three spaces. ${ }^{4-6}$

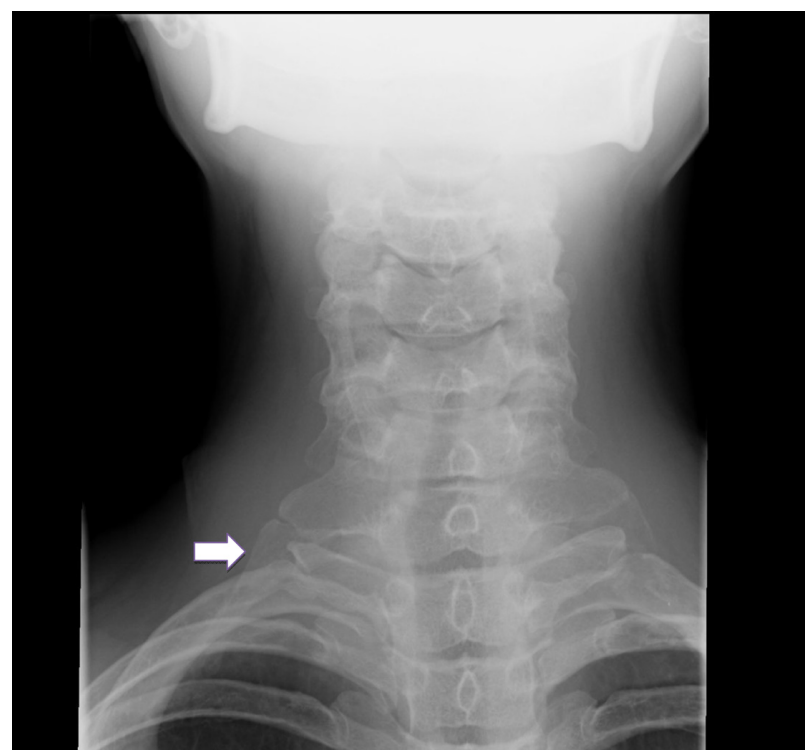

Figure 2 Frontal chest radiograph (cropped) of a I5-year-old girl with intermittent paresthesia.

Note: Swelling of her right arm demonstrates a typical right-sided cervical rib (arrow).

Dynamic narrowing does not affect the interscalene triangle but does narrow the costoclavicular, coracopectoral, or retropectoral spaces. However, retropectoral space narrowing is not commonly manifested clinically as vascular TOS, despite evidence of compression of the vascular structures. ${ }^{7}$ Involvement of the retropectoral space is common in neurogenic TOS, with a recent study showing involvement in some form in $50 \%$ of all TOS cases, even if the primary site of entrapment is not at this site. ${ }^{8}$ The costoclavicular space is the most common site for vascular $\operatorname{TOS}^{9}$ whereas neurologic al TOS (NTOS) is equally frequent in both the interscalene space and costoclavicular space. ${ }^{9}$

Direct compression of neurovascular structures in the thoracic outlet may be due to extrinsic compression. Soft tissue abnormalities resulting in TOS can be congenital or acquired.

Congenital soft tissue abnormalities include variation in scalene muscle insertions, hypertrophy of the scalene muscles, or accessory scalene muscles. Scalene muscle variation can affect nerve roots of the brachial plexus as they emerge between the anterior and middle scalene. ${ }^{10-12}$ An accessory scalene muscle or scalenus minimus may vary in its origin from $\mathrm{C} 6$ or $\mathrm{C} 7$ and inserts into the scalene tubercle of the first rib or cervical pleura of the lung and this can also cause TOS. ${ }^{12-14}$ Anomalies such as fibromuscular bands extending between the transverse process of a cervical vertebra and the first thoracic rib were described by Roos. ${ }^{13}$

Acquired abnormalities may be the result of trauma, resulting in fibrosis around the neurovascular tissue. Histological changes can be detected within the paraneurium, which is 
thought to result in a "pain-immobility-fibrosis loop", an important step in the development of this condition. ${ }^{15}$ Fibrosis can be the result of a single traumatic event resulting in fracture or tissue injury, or can be secondary to repetitive injury to muscle and ligaments resulting from normal movements. ${ }^{16}$ The latter is an important cause of TOS and can be underdiagnosed if a full clinical history is not obtained. Hypertrophy of the normal scalene muscle can result in narrowing of the interscalene space resulting in TOS, as can hypertrophy of the pectoralis minor tendon, but with narrowing seen in the retropectoral space. ${ }^{17,18}$ These two conditions can occur in athletes with no other anatomical abnormalities.

The current classification of TOS is based upon the structures involved.

\section{Vascular TOS}

This syndrome can be subdivided into arterial and venous TOS.

\section{Arterial vascular TOS}

This is the least common form of TOS, accounting for less than $1 \%$ of all cases. ${ }^{1}$ Arterial vascular TOS is the result of compression of the subclavian artery at the root of the neck, most commonly in the costoclavicular space, for example, by an accessory cervical rib, fibrous band, or deformed thoracic rib. Arterial aneurysmal dilation may develop distal to the point of obstruction because of the turbulent flow resulting from proximal narrowing. Thrombus can form in the aneurysmal sac and may occasionally propagate distally, resulting in an acutely ischemic upper limb. Primary symptoms are paresthesia, coldness, and numbness of the limb because of vasa nervosa insufficiency causing ischemic neuritis of the brachial plexus. Acute ischemia presents as an acutely painful limb.

\section{Venous vascular TOS}

Primary spontaneous thrombosis of subclavian or axillary vein occurs most commonly in young men and symptoms include swelling, heaviness, and dilation of superficial veins. Sometimes a history of repetitive motion or severe exertion can be elicited. ${ }^{19}$ Thrombosis of the subclavian or axillary vein results in an acutely swollen, painful upper limb. This is also known as "Paget-von Schroetter"2 disease or "effort thrombosis syndrome".

\section{NTOS}

This is often subdivided into true NTOS and disputed, or nonspecific, TOS.

\section{True NTOS}

Neurological symptoms can be secondary to compression of the nerve roots of the brachial plexus within the thoracic outlet. Raynaud's phenomenon may occur as a consequence of sympathetic overstimulation due to involvement of the lower trunks of the brachial plexus which carry sympathetic fibers. ${ }^{16}$ An anatomical abnormality such as a fibrous band extending from the transverse process of $\mathrm{C} 7$ to the first rib results in stretching of the proximal fibers of the lower trunk of the brachial plexus. Patients may complain of nonspecific paresthesia or pain in the arm or hand and motor weakness of muscles of hand and forearm, with or without atrophy of the thenar eminence. Some patients may present with pain in the chest or neck or even with headache. ${ }^{20}$ Symptoms may often have a chronic course and the patient may not seek a medical opinion for years. The interscalene triangle is more frequently found to be narrowed in patients with TOS than in asymptomatic patients, ${ }^{21}$ although NTOS is equally likely to result from compression arising in the interscalene space or costoclavicular space. ${ }^{9}$

\section{Disputed or nonspecific TOS}

Some authors have proposed the category of disputed or nonspecific TOS for situations in which there is a degree of overlap between neurogenic and vascular TOS. ${ }^{22,23}$ Other authors have argued that neurogenic TOS is wrongly labeled as vascular TOS in many patients. ${ }^{1}$ More recently, the use of the term "disputed TOS" has been discouraged due to its ambiguity.

NTOS and nonspecific TOS are the most common forms of TOS and are often associated with a history of trauma. ${ }^{1}$

\section{Clinical diagnosis}

Diagnosing TOS is challenging, as symptoms can be chronic and overlap with other presentations. Patients may present with pain, paresthesia, weakness, claudication, and muscle wasting in the upper limb, along with possible neck pain, chest pain, headache, vertigo, and dizziness. ${ }^{24} \mathrm{~A}$ minority of patients will present with specific findings, such as a GilliattSumner hand, which is characterized by severe atrophy of the abductor pollicis brevis muscle, with severe atrophy of the interosseous and hypothenar muscles of the same side. ${ }^{25}$ A variety of dynamic provocation tests can reproduce the symptoms from TOS. ${ }^{26}$ These tests can help the clinician to exclude other pathologies. Some of the most widely used tests are as follows.

\section{Adson's test}

The patient's radial artery is palpated while the patient is seated in a chair, with the arm by the side of the patient. 
The patient is then asked to take long deep breaths with their face turned toward the affected arm. If the radial pulse is absent or deficient, the test is regarded as positive. Many authors do not favor this test as positive results can be elicited in healthy, asymptomatic individuals. ${ }^{27}$

\section{Wright's test}

Wright's test is also performed in the sitting position. The patient's arm is slowly abducted while the radial artery is being palpated. A loss of pulse confirms subclavian or axillary nerve compression in the thoracic outlet during abduction or overhead abduction of the arm.

\section{Roos' test or elevated arm stress test}

The patient is asked to open and close the fist rapidly with $90^{\circ}$ of shoulder abduction and $90^{\circ}$ of elbow flexion. This is typically performed for 1 minute or 3 minutes to assess reproduction of symptoms. Tingling or numbness while opening and closing the hands in this position supports the clinical diagnosis of TOS. Inability to complete this test due to pain has a low false-positive rate in neurogenic TOS. ${ }^{28}$

\section{Military brace test}

The test is performed in the standing position with the examiner standing behind the patient. The examiner palpates the radial artery while the patient's shoulder is extended, with the elbow extended. Loss of the radial pulse confirms compression of subclavian artery at the thoracic outlet.

\section{Upper limb tension test}

A modified version of Elvey's upper limb tension test is described by Sanders et al. ${ }^{1}$ First the arm is abducted to $90^{\circ}$ with the elbow extended. Second, the wrist is dorsiflexed and, finally, the head is tilted to one side, ear to shoulder. While positions 1 and 2 elicit symptoms on the ipsilateral side, position 3 elicits symptoms on the contralateral side. Pain or paresthesia is a positive response. The strongest positive test is onset of symptoms in position 1, with increased symptoms in the other two positions. Sanders et al note that a positive upper limb tension test is not pathognomonic of NTOS, but does indicate proximal compression of the nerve roots or brachial plexus. ${ }^{1}$

Although these tests may be used at initial clinical assessment, they all have high false-positive rates.

\section{Scalene and pectoralis minor blocks}

The use of anesthetic blocks of the anterior scalene and pectoralis minor muscles, both as diagnostic and therapeutic maneuvers, has increased in recent years. There have been no randomized controlled studies to assess this technique, but there are many reports of its use..$^{29-31}$

Intramuscular injection can be performed either clinically or with image guidance. Typically, $1 \%$ lidocaine is utilized. Clinically guided injection can be performed in areas of tenderness, with care taken to avoid the pleural space. Imageguided techniques have been described using ultrasound ${ }^{29}$ and $\mathrm{CT} \cdot{ }^{30}$ Intramuscular injection under ultrasound guidance has been shown to be safe and well tolerated. ${ }^{31}$

Patients with a good response to local anesthetic block have been shown to have better outcomes following surgery compared to those who have not. ${ }^{32}$ The use of local anesthetic blocks has been shown to be of particular use in directing therapeutic options for recurrent TOS. ${ }^{8}$

\section{Electrophysiology}

The use of electromyography and nerve conduction studies in diagnosing TOS is controversial. Rousseff et al found electrophysiological studies unhelpful in establishing the diagnosis of TOS, ${ }^{33}$ while others have reported success both in diagnosis and assessing response to treatment. ${ }^{32}$ These tests are not necessarily part of the routine work-up of a patient with suspected TOS, as they provide information about only the neurological structures that pass through the thoracic outlet. However, they may be useful when performed in patients with nonspecific neurological symptoms because they can distinguish myogenic and neurogenic causes. If there is a neurogenic abnormality, the neurological level affected can be identified. In neurogenic TOS, in which brachial plexus injury can occur at various levels between the neck and axilla and to varying degree, electromyography can be used to assess the neurological compromise and level of injury to the brachial plexus. Findings include reduced amplitudes in the median motor, ulnar sensory, and medial antebrachial cutaneous nerves and suggest chronic or prolonged compression. The collection of findings has been shown to have a high sensitivity for neurogenic TOS. ${ }^{34}$

Electrophysiological studies can be of use in excluding non-neurological causes of symptoms, when considering neurogenic TOS as diagnosis. ${ }^{35}$ They are also valuable in establishing reproducible objective data in diagnosing the extent of damage to nerves and recovery following surgery. ${ }^{32,36}$

Electrophysiological studies are therefore not necessarily routine, but may be beneficial in patients with suspected neurogenic TOS, when supported by appropriate clinical examination and radiological investigations. 


\section{Radiography of the cervical spine and chest}

$\mathrm{X}$-ray of the cervical spine and chest are the first imaging investigations for TOS. Skeletal abnormalities including cervical rib, exostosis, callus formation, bony spur, or a calcified fibrous ligament can be seen on a plain radiograph. Cervical ribs are present in $1 \%$ of the population and are a radiographic finding in 5\%-10\% of patients with TOS. ${ }^{37,38}$ It is also useful to exclude an obvious pulmonary apical tumor as a cause of TOS-like symptoms.

The decision to investigate further will depend upon the presentation, history, and clinical findings.

\section{Digital subtraction angiography (DSA)}

DSA has been regarded as the gold standard investigation for arterial abnormalities. ${ }^{39} \mathrm{In}$ modern practice, DSA is often only used in combination with interventional techniques to institute treatment or refine a particularly difficult diagnosis. DSA has been employed to demonstrate obstruction to flow in the subclavian, axillary, or brachial arteries. The initial assessment of these patients is now generally performed with CT angiography (CTA) or magnetic resonance angiography (MRA) to avoid the risks of arterial puncture, including lifethreatening hemorrhage, dissection, thrombosis, hematoma, and pseudoaneurysm formation. ${ }^{40} \mathrm{DSA}$ can be combined with thrombolytic therapy in patients with arterial thrombus or embolus, enabling thrombolysis and restoration of blood flow to the affected limb. Catheter-directed or mechanical thrombolysis can also be used to treat acute venous occlusion.

Soft tissue abnormalities can potentially be diagnosed indirectly by demonstrating filling defects or extrinsic compression of the imaged vascular structures, although crosssectional imaging is often more useful because it allows direct assessment of these structures. DSA can demonstrate abnormalities such as arterial aneurysm or dissection (Figure 3), although the presence of thrombus limits this.

Modern cross-sectional imaging techniques are very effective in demonstrating vascular pathologies such as impingement or obstruction ${ }^{41,42}$ and have the advantage of being much less invasive.

\section{Ultrasound scan (USS)}

USS is infrequently used as the sole modality for diagnosing TOS. The use and availability of ultrasound is highly variable. USS is dynamic and can be combined with clinical examination techniques to provide further evidence in support of a diagnosis of TOS. USS has been used to assist in

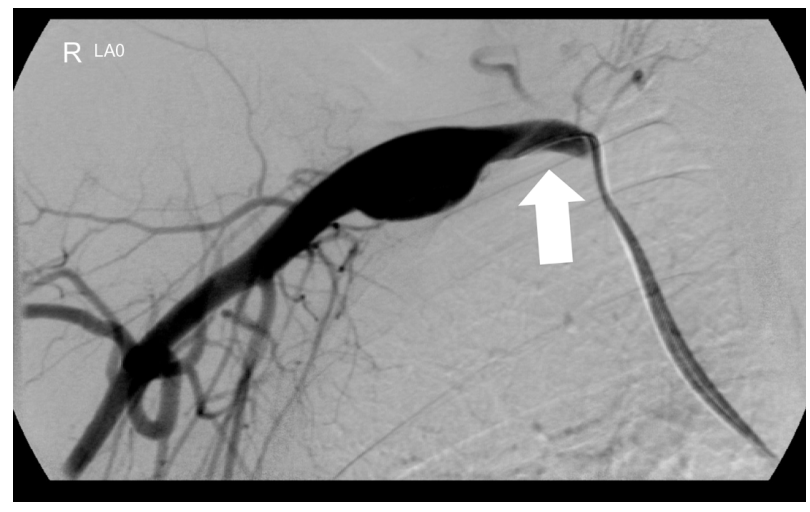

Figure 3 Digital subtraction angiography of a 24-year-old male who presented with an acutely ischemic right arm.

Notes: Clinical examination suggested a supraclavicular aneurysm. Arch aortography and selective arteriography of the right subclavian artery confirmed a fusiform aneurysm of the subclavian artery and a proximal fibrous band in keeping with compression in the thoracic outlet. There was a long-segment occlusion of the brachial artery, due to recent embolization of thrombus from the aneurysm.

making the diagnosis of NTOS at the interscalene hiatus. Dynamic assessment of the brachial plexus at the level of the interscalene hiatus using ultrasound while performing clinical tests can demonstrate compression between anterior and middle scalene muscles. ${ }^{43}$ Simultaneous provocation of symptoms with this ultrasonographic appearance can confirm the diagnosis of compression of the brachial plexus.

Ultrasonography is very useful in diagnosing axillary or subclavian vein thrombosis in Paget-von Schroetter syndrome and has a high diagnostic accuracy with appropriately experienced operators (Figure 4) ${ }^{44}$ However, use of ultrasound is limited in diagnosing other forms of TOS.

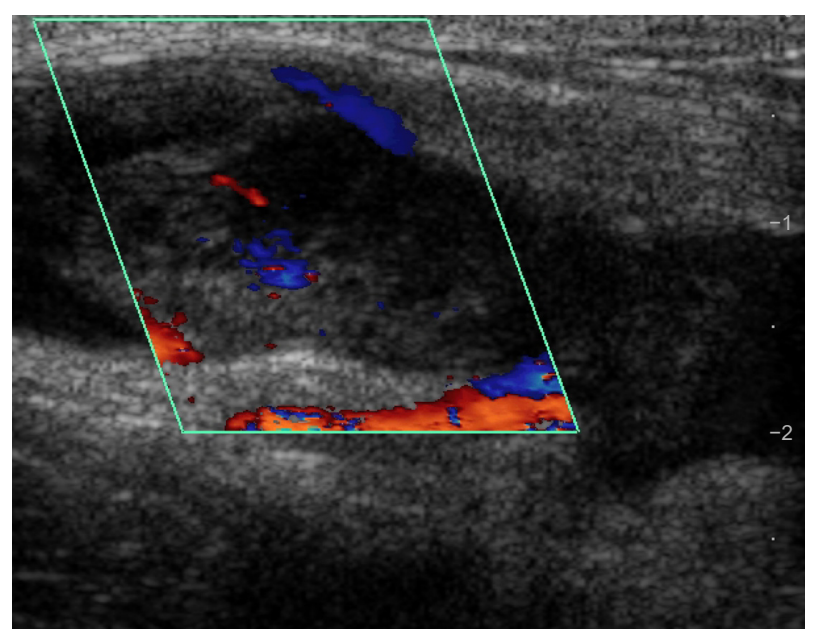

Figure 4 Ultrasound of the right subclavian vein in a 17-year-old male. Notes: The patient presented the day following trampolining and heavy lifting with pain and swelling in his right arm and distended superficial veins on the right side of the chest. The study demonstrates a mixed echogenicity filling defect within a dilated subclavian vein, in keeping with acute thrombosis (Paget-von Schroetter syndrome). 
The role of USS in the assessment of suspected arterial TOS is controversial and not well established because of overlap between normal variation and pathological appearances. Stapleton et $\mathrm{al}^{45}$ demonstrated complete lack of blood flow in abduction and external rotation of the arm in healthy individuals.

\section{CT and CTA}

$\mathrm{CT}$ is a noninvasive technique which uses ionizing radiation to create cross-sectional images. It is fast and widely available and often useful in the assessment of a patient with an acute presentation. CTA is frequently used in patients with acute ischemia of the upper limb, as the extent of the arterial occlusion can be demonstrated at the same time as any potential proximal causes of embolus such as arterial aneurysms. CTA requires intravenous access and contrast administration.

CT allows multiplanar visualization of the thoracic inlet and can be combined with angiographic techniques to assess compression of both the artery and the vein. CT images can be manipulated on a workstation to show the required length of the vessel on a single image (Figure 5), or combined with three-dimensional reconstructions to assist with surgical planning. Three-dimensional reconstructions provide an overview of the bony and contrast-filled structures and can be created in any plane. Non-contrast multi-detector CT of the neck and upper chest can be performed to describe the anatomy of the thoracic outlet followed by elevated arm CTA to demonstrate dynamic compression. ${ }^{5}$

CTA can be performed with the patient prone with the arm abducted over the head with an injection of $100 \mathrm{~mL}$

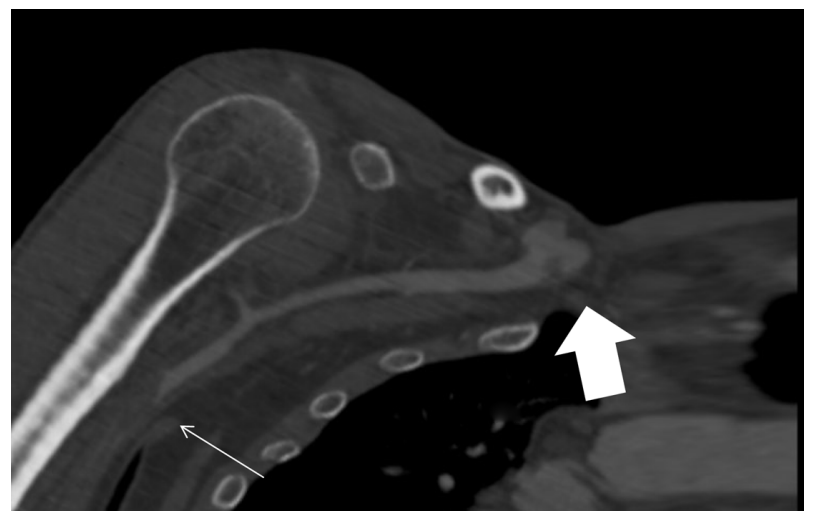

Figure 5 Curved multiplanar reconstruction of a computed tomography study in a patient who presented with acute ischemia of the right arm.

Notes: Note the aneurysm of the subclavian artery (large arrow), which was present immediately distal to the cervical rib causing thoracic outlet syndrome. The brachial artery terminates abruptly with low attenuation material within the lumen consistent with thrombus from the aneurysm propagating down the arm (small arrow). This patient was treated with catheter-directed thrombolysis and subsequent resection of the cervical rib.

Abbreviation: CT, computed tomography. intravenous iodinated contrast at $4-5 \mathrm{~mL} / \mathrm{s}$ and either a bolus-tracking triggered acquisition or a delay of 15-20 seconds from the start of the injection. For patients unable to lie prone, supine imaging with raised arm prior to the CTA acquisition is often satisfactory. Optimum results are achieved by injecting intravenous contrast on the asymptomatic side, so as to minimize beam hardening artifact from the subclavian vein opacification. Images should be viewed using both soft tissue windows (window length: 400, window width: 40) and vascular windows (window length: 650, window width: 220).

Limitations of CTA include exposure of the patient to ionizing radiation and the risks of intravenous contrast injection, such as anaphylactoid reactions and nephrotoxicity in vulnerable patients. CTA is useful in establishing the vascular TOS resulting from bone or soft tissue compression.

CT has limited soft tissue contrast resolution and is less successful at demonstrating the interscalene hiatus, limiting its usefulness in NTOS. ${ }^{21}$

\section{Magnetic resonance imaging (MRI)}

MRI is increasingly used in the diagnosis of vascular TOS. MRI has the advantages of being a nonionizing radiation technique and gives excellent soft tissue resolution, which helps to demonstrate soft tissue causes of TOS. MRA techniques can provide subtracted angiography.

The range of abnormalities that can be seen with MRI includes accessory muscles (scalenus minimus, subclavius

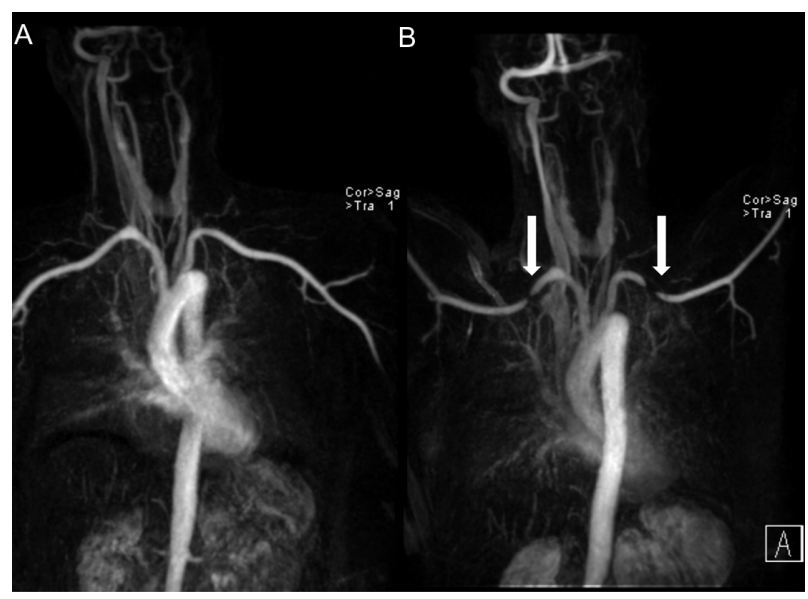

Figure $63 \mathrm{D}$ rotation dynamic MRA sequence.

Notes: A 38-year-old female presented with intermittent pain and numbness in her fingers, exacerbated by certain movements. The images show a subtracted threedimensional rotational MIP of a contrast-enhanced MRA sequence with (A) arms down and (B) arms raised. Severe compression of the subclavian artery can be seen on both sides (arrows).

Abbreviations: CT, computed tomography; MRA, magnetic resonance angiography; MIP, maximum intensity projection. 
posticus, duplicated omohyoid inferior belly, pectoralis minimus muscle) and muscle hypertrophy (omohyoid inferior belly, pectoralis muscle, scalene, and subclavius) as well as fibrous bands. ${ }^{9}, 14,16,46$

Typical anatomical sequences used would include fast spin echo T1 sagittal high-resolution imaging, which can be combined with $\mathrm{T} 2$ fat saturation or short tau inversion recovery coronal sequences. Short tau inversion recovery sequences take a little longer to acquire but provide more uniform fat suppression over a large field of view.

MRA datasets are acquired before contrast administration and then during arterial and venous phases. An intravenous gadolinium agent is given by infusion through a peripheral venous cannula. A dose of $0.1 \mathrm{mmol} / \mathrm{kg}$ is given with the arms raised, and this dose can be repeated with the arms lowered.

MRA sequences are obtained with the arms abducted and raised above the shoulders and later with arms by the sides. This can demonstrate compression of the vascular structures with abduction and reversal of these findings with the arms lowered. Vascular abnormalities are well demonstrated using rotated maximum intensity projections (Figure 6).

Blood pool contrast agents, which are retained in the intravascular space because of binding to albumin, can be used to assess the arterial and venous structures following a single, lower-dose injection. ${ }^{41}$ This enables higher-resolution imaging because the contrast is not rapidly excreted after the first pass and images can be acquired over a greater length of time in the steady state.

There is still controversy over the use of MRI for neurogenic TOS. There are newer techniques such as 3T magnetic

Table I Advantages and disadvantages of different imaging modalities

\begin{tabular}{lll}
\hline Modality & Advantages & Disadvantages \\
\hline Plain radiography & Widely available & $\begin{array}{l}\text { Only useful for bony } \\
\text { abnormalities }\end{array}$ \\
Ultrasound scan & $\begin{array}{l}\text { Dynamic } \\
\text { Nonionizing radiation }\end{array}$ & $\begin{array}{l}\text { Operator dependent } \\
\text { Limited usefulness }\end{array}$ \\
& & other than assessing \\
Computed & High spatial resolution & Radiation dose \\
tomography & Can be combined with & Limited soft tissue \\
Magnetic & angiographic techniques & contrast \\
resonance imaging & Excellent soft tissue & Time-consuming \\
& contrast & Expensive \\
& Dynamic angiographic & Contraindications \\
& techniques & in some patients \\
Can be combined with & Radiation dose \\
angiography & interventional procedure & Invasive \\
& (eg, in venous occlusion) & Risk of complications \\
& & Limited diagnostic use \\
\hline
\end{tabular}

resonance neurography (high-resolution imaging), which can enable identification of fibrous bands impinging on the lower brachial plexus ${ }^{47}$ although this technique is not widely used in clinical practice.

The advantages and disadvantages of the various imaging modalities are summarized in Table 1.

\section{Conclusion}

"Thoracic outlet syndrome" is a term that covers a range of abnormalities causing a variety of symptoms. Appropriate investigations need to be tailored to the individual and there is no single diagnostic test. Clinical history and examination dictates the need for electrophysiological testing and subsequent imaging. A chest radiograph allows identification of bony abnormalities and apical lung tumors. Ultrasonography is useful in cases of suspected acute thrombosis of the upper limb. CT combined with CTA provides a useful overview of the anatomical structures and vessel compression in abduction, although is probably more appropriately used in emergency situations or where there are difficulties or contraindications to performing MRI. MRI and MRA allow dynamic assessment of the thoracic outlet, which is most helpful in delineating the cause and level of arterial compression in arterial TOS.

\section{Disclosure}

The authors report no conflicts of interest in this work.

\section{References}

1. Sanders RJ, Hammond SL, Rao NM. Diagnosis of thoracic outlet syndrome. J Vasc Surg. 2007;46(3):601-604.

2. Peet RM, Henriksen JD, Anderson TP, Martin GM. Thoracic-outlet syndrome: evaluation of a therapeutic exercise program. Proc Staff Meet Mayo Clin. 1956;31(9):281-287.

3. Samarasam I, Sadhu D, Agarwal S, Nayak S. Surgical management of thoracic outlet syndrome: a 10-year experience. ANZ J Surg. 2004;74(6):450-454

4. Remy-Jardin M, Doyen J, Remy J, Artaud D, Fribourg M, Duhamel A. Functional anatomy of the thoracic outlet: evaluation with spiral CT. Radiology. 1997;205(3):843-851.

5. Matsumura JS, Rilling WS, Pearce WH, Nemcek AA Jr, Vogelzang RL, Yao JS. Helical computed tomography of the normal thoracic outlet. J Vasc Surg. 1997;26(5):776-783.

6. Remy-Jardin M, Remy J, Masson P, et al. Helical CT angiography of thoracic outlet syndrome: functional anatomy. AJR Am J Roentgenol. 2000;174(6):1667-1674.

7. Demirbag D, Unlu E, Ozdemir F, et al. The relationship between magnetic resonance imaging findings and postural maneuver and physical examination tests in patients with thoracic outlet syndrome: results of a double-blind, controlled study. Arch Phys Med Rehabil. 2007;88(7):844-851.

8. Sanders RJ. Recurrent neurogenic thoracic outlet syndrome stressing the importance of pectoralis minor syndrome. Vasc Endovascular Surg. 2011;45(1):33-38.

9. Demondion X, Bacqueville E, Paul C, Duquesnoy B, Hachulla E, Cotten A. Thoracic outlet: assessment with MR imaging in asymptomatic and symptomatic populations. Radiology. 2003;227(2):461-468. 
10. Poitevin LA. Thoraco-cervico-brachial confined spaces an anatomic study. Ann Chir Main. 1988;7(1):5-13. English, French.

11. Luoma A, Nelems B. Thoracic outlet syndrome. Thoracic surgery perspective. Neurosurg Clin N Am. 1991;2(1):187-226.

12. Lawson FL, McKenzie KG. The scalenus minimus muscle. Can Med Assoc J. 1951;65(4):358-361.

13. Roos DB. Congenital anomalies associated with thoracic outlet syndrome. Anatomy, symptoms, diagnosis, and treatment. Am J Surg. 1976;132(6):771-778.

14. Brantigan CO, Roos DB. Diagnosing thoracic outlet syndrome. Hand Clin. 2004;20(1):27-36.

15. Crotti FM, Carai A, Carai M, Sgaramella E, Sias W. Post-traumatic thoracic outlet syndrome (TOS). Acta Neurochir Suppl. 2005;92:13-15.

16. Aralasmak A, Cevikol C, Karaali K, et al. MRI findings in thoracic outlet syndrome. Skeletal Radiol. 2012;41(11):1365-1374.

17. Baltopoulos P, Tsintzos C, Prionas G, Tsironi M. Exercise-induced scalenus syndrome. Am J Sports Med. 2008;36(2):369-374.

18. Nichols AW. The thoracic outlet syndrome in athletes. J Am Board Fam Pract. 1996;9(5):346-355.

19. Flinterman LE, Van Der Meer FJM, Rosendaal FR, Doggen CJ. Current perspective of venous thrombosis in the upper extremity. $J$ Thromb Haemost. 2008;6(8):1262-1266.

20. Raskin NH, Howard MW, Ehrenfeld WK. Headache as the leading symptom of the thoracic outlet syndrome. Headache. 1985;25(4):208-210.

21. Demondion X, Herbinet P, Van Sint Jan S, Boutry N, Chantelot C, Cotten A. Imaging assessment of thoracic outlet syndrome. Radiographics. 2006;26(6):1735-1750.

22. Landry GJ, Moneta GL, Taylor LM Jr, Edwards JM, Porter JM. Long-term functional outcome of neurogenic thoracic outlet syndrome in surgically and conservatively treated patients. J Vasc Surg. 2001;33(2):312-317; discussion 317-319.

23. Urschel JD, Hameed SM, Grewal RP. Neurogenic thoracic outlet syndromes. Postgrad Med J. 1994;70(829):785-789.

24. Fitzgerald G. Thoracic outlet syndrome of pectoralis minor etiology mimicking cardiac symptoms on activity: a case report. J Can Chiropr Assoc. 2012;56(4):311-315.

25. Gilliatt RW, Le Quesne PM, Logue V, Sumner AJ. Wasting of the hand associated with a cervical rib or band. J Neurol Neurosurg Psychiatry. 1970;33(5):615-624.

26. Sanders RJ, Hammond SL, Rao NM. Thoracic outlet syndrome: a review. Neurologist. 2008;14(6):365-373.

27. Gergoudis R, Barnes RW. Thoracic outlet arterial compression: prevalence in normal persons. Angiology. 1980;31(8):538-541.

28. Plewa MC, Delinger M. The false-positive rate of thoracic outlet syndrome shoulder maneuvers in healthy subjects. Acad Emerg Med. 1998;5(4):337-342.

29. Spence BC, Beach ML, Gallagher JD, Sites BD. Ultrasound-guided interscalene blocks: understanding where to inject the local anaesthetic. Anaesthesia. 2011;66(6):509-514.

30. Mashayekh A, Christo PJ, Yousem DM, Pillai JJ. CT-guided injection of the anterior and middle scalene muscles: technique and complications. AJNR Am J Neuroradiol. 2011;32(3):495-500.
31. Torriani M, Gupta R, Donahue DM. Sonographically guided anesthetic injection of anterior scalene muscle for investigation of neurogenic thoracic outlet syndrome. Skeletal Radiol. 2009;38(11):1083-1087.

32. Jordan SE, Machleder HI. Diagnosis of thoracic outlet syndrome using electrophysiologically guided anterior scalene blocks. Ann Vasc Surg. 1998;12(3):260-264.

33. Rousseff R, Tzvetanov P, Valkov I. Utility (or futility?) of electrodiagnosis in thoracic outlet syndrome. Electromyogr Clin Neurophysiol. 2005;45(3):131-133.

34. Kothari MJ, Macintosh K, Heistand M, Logigian EL. Medial antebrachial cutaneous sensory studies in the evaluation of neurogenic thoracic outlet syndrome. Muscle Nerve. 1998;21(5):647-649.

35. Gillard J, Pérez-Cousin M, Hachulla E, et al. Diagnosing thoracic outlet syndrome: contribution of provocative tests, ultrasonography, electrophysiology, and helical computed tomography in 48 patients. Joint Bone Spine. 2001;68(5):416-424.

36. Passero S, Paradiso C, Giannini F, Cioni R, Burgalassi L, Battistini N. Diagnosis of thoracic outlet syndrome. Relative value of electrophysiological studies. Acta Neurol Scand. 1994;90(3):179-185.

37. White PW, Fox CJ, Feuerstein IM. Cervical rib causing arterial thoracic outlet syndrome. J Am Coll Surg. 2009;209(1):148-149.

38. Chang KZ, Likes K, Davis K, Demos J, Freischlag JA. The significance of cervical ribs in thoracic outlet syndrome. J Vasc Surg. 2013;57(3):771-775.

39. François CJ, Carr JC. MRI of the thoracic aorta. Cardiol Clin. 2007;25(1):171-184, vii.

40. Das R, Ahmed K, Athanasiou T, Morgan RA, Belli AM. Arterial closure devices versus manual compression for femoral haemostasis in interventional radiological procedures: a systematic review and meta-analysis. Cardiovasc Intervent Radiol. 2011;34(4):723-738.

41. Lewis M, Yanny S, Malcolm PN. Advantages of blood pool contrast agents in MR angiography: a pictorial review. J Med Imaging Radiat Oncol. 2012;56(2):187-191.

42. Kock MC, Adriaensen ME, Pattynama PM, et al. DSA versus multidetector row $\mathrm{CT}$ angiography in peripheral arterial disease: randomized controlled trial. Radiology. 2005;237(2):727-737.

43. Fried SM, Nazarian LN. Dynamic neuromusculoskeletal ultrasound documentation of brachial plexus/thoracic outlet compression during elevated arm stress testing. Hand (N Y). 2013;8(3):358-365.

44. Klitfod L, Broholm R, Baekgaard N. Deep venous thrombosis of the upper extremity. A review. Int Angiol. 2013;32(5):447-452.

45. Stapleton C, Herrington L, George K. Sonographic evaluation of the subclavian artery during thoracic outlet syndrome shoulder manoeuvres. Man Ther. 2009;14(1):19-27.

46. Aralasmak A, Karaali K, Cevikol C, Uysal H, Senol U. MR imaging findings in brachial plexopathy with thoracic outlet syndrome. AJNR Am J Neuroradiol. 2010;31(3):410-417.

47. Baumer P, Kele H, Kretschmer T, et al. Thoracic outlet syndrome in 3T MR neurography-fibrous bands causing discernible lesions of the lower brachial plexus. Eur Radiol. 2014;24(3):756-761.
Journal of Vascular Diagnostics

\section{Publish your work in this journal}

Journal of Vascular Diagnostics is an international, peer-reviewed journal of diagnostics, focusing on non invasive vascular investigation methods involved in the evaluation of vascular diseases. The journal is committed to the rapid publication in the fields of vascular diseases. Original research, review, case reports, expert opinion and commentaries

\section{Dovepress}

are all considered for publication. The manuscript management system is completely online and includes a very quick and fair peer-review system, which is all easy to use. Visit http://www.dovepress.com/testimonials.php to read real quotes from published authors. 